\title{
Laboratory monitoring to reduce adverse drug-related events: a mixed methods study
}

\author{
Teresa N Harrison, SM; Kristi Reynolds, PhD, MPH; Erin E Hahn, PhD, MPH; Corrine E Munoz-Plaza, MPH; \\ David K Yi, MPH; Heidi Fischer, PhD; Tiffany Q Luong, MPH; John J Sim, MD; Jeffrey Brettler, MD; Joel Handler, MD; \\ Brian S Mittman, PhD; Hardeep Singh, MD, MPH; Michael H Kanter, MD; and Kim N Danforth, ScD, MPH
}

\section{What is already known about this subject} potassium and creatinine for patients on angiotensin-converting enzyme inhibitors (ACEIs) or angiotensin receptor blockers (ARBs) can reduce preventable adverse drug events.

- Lack of annual laboratory monitoring may result in delayed diagnosis of clinically important sequelae, yet studies have found that a substantial number of patients do not receive the recommended laboratory tests.
- Annual monitoring of serum

\section{What this study adds}

- This analysis found that $9 \%$ of patients taking ACEls or ARBs did not complete recommended annual laboratory monitoring for adverse drug-related events.

- Factors associated with a reduced risk of a care gap included older age, Asian or Hispanic ethnicity, having several morbidities, and having a cardiologist or nephrologist as the prescribing provider of the ACEI or ARB.

- Monitoring strategies could show a substantial benefit in reducing adverse drug events by increasing compliance with recommended laboratory testing.

\section{ABSTRACT}

BACKGROUND: Therapy with angiotensinconverting enzyme inhibitors (ACEIs) and angiotensin receptor blockers (ARBs) requires laboratory monitoring to avoid hyperkalemia and acute kidney failure.

OBJECTIVE: To assess the frequency of recommended annual serum potassium and creatinine monitoring and determine potential factors associated with care gaps among adults dispensed an ACEI or ARB.

METHODS: This mixed-methods study integrated findings from a retrospective cohort study and individual patient interviews. Adults aged 21 years and over within Kaiser Permanente Southern California with at least 180 treatment days of an ACEI and/or ARB in 2015 were included. Patients invited for qualitative interviews included those who did and did not complete the recommended laboratory tests. We assessed the proportion of patients completing both recommended laboratory tests, factors associated with not receiving laboratory monitoring, and patients' insights into barriers and facilitators of recommended monitoring.

RESULTS: Of 437,544 patients who received an ACEI or ARB, $9.0 \%$ did not receive both a serum potassium and creatinine laboratory test during treatment (defined as a care gap). Lower risk of a care gap was observed for patients with increasing age (rate ratio $[R R]$ per 10 -year increase $=0.78,95 \%$ $\mathrm{Cl}=0.77-0.79)$; diabetes mellitus ( $\mathrm{RR}=0.62$, $95 \% \mathrm{Cl}=0.60-0.64)$; hypertension $(\mathrm{RR}=0.71$, $95 \% \mathrm{Cl}=0.71-0.74)$; Charlson Comorbidity Index score of at least 2 ( $R R=0.62,95 \%$ $\mathrm{Cl}=0.60-0.64)$; those who changed medication classes $(\mathrm{RR}=0.53,95 \% \mathrm{Cl}=0.51-0.56)$; and patients with a cardiologist $(R R=0.81$, $95 \% \mathrm{Cl}=0.73-0.90)$ or nephrologist $(\mathrm{RR}=0.60$, $95 \% \mathrm{Cl}=0.52-0.69)$ as their prescribing provider. Twenty-five patients completed the

\section{Author affiliations}

Teresa N Harrison, SM; Erin E Hahn, PhD, $\mathrm{MPH}$; Corrine E Munoz-Plaza, MPH; David K Yi, MPH; Heidi Fischer, PhD; Tiffany Q Luong, MPH; and Brian S Mittman, PhD, Department of Research \& Evaluation, Kaiser Permanente Southern California, Pasadena. Kristi Reynolds, PhD, MPH, Department of Research \& Evaluation, Kaiser Permanente Southern California, and Department of Health Systems Science, Kaiser Permanente Bernard J Tyson School of Medicine, Pasadena, CA. John J Sim, MD, Division of Nephrology and Hypertension, Kaiser Permanente Los Angeles Medical Center, Los Angeles, CA. Jeffrey Brettler, MD, Kaiser Permanente West Los Angeles Medical Center, Los Angeles, CA. Joel Handler, MD, Southern California Permanente Medical Group, Yorba Linda. Hardeep Singh, MD, $\mathrm{MPH}$, Center for Innovations in Quality, Effectiveness and Safety, Michael E DeBakey VA Medical Center; Department of Medicine, Baylor College of Medicine, Houston, TX. Michael H Kanter, MD, Department of Clinical Science, Kaiser Permanente Bernard J. Tyson School of Medicine, Pasadena, CA. Kim N Danforth, ScD, MPH, Department of Research and Evaluation, Kaiser Permanente Southern California, Pasadena.

AUTHOR CORRESPONDENCE:

Teresa N Harrison, 626.564.3604;

Teresa.N.Harrison@kp.org

J Manag Care Spec Pharm 2022;28(1):16-25

Copyright $@ 2022$, Academy of Managed Care Pharmacy. All rights reserved. 
qualitative interviews. Patients often lacked knowledge about the need for laboratory monitoring, cited logistical barriers to accessing the laboratory, and deemed the reminders they received through an outpatient safety program as a facilitator to completing tests.

CONCLUSIONS: Given the large patient population on ACEI and ARB medications, monitoring and support strategies such as electronic clinical surveillance could be important in addressing care gaps and potentially reducing adverse drug effects.

Angiotensin-converting enzyme inhibitors (ACEIs) and angiotensin receptor blockers (ARBs) are commonly prescribed medications for patients with hypertension, heart failure, and chronic kidney disease, including diabetic kidney disease. ${ }^{1-3}$ Over 674 million antihypertensive prescriptions (including 114 million for lisinopril and 54 million for losartan) were dispensed in 2018, making them the most commonly prescribed medications in the United States. ${ }^{4}$ Adverse effects of these medications may include hyperkalemia and increased serum creatinine levels, ${ }^{5-7}$ which, if untreated, may lead to more serious adverse events. Use of ACEIs or ARBs by adults taking antihypertensive medications has been associated with a 54\% and 7\% increased risk of hyperkalemia, respectively. ${ }^{8}$

A study of patients initiating ACEIs within the Veteran's Affairs Healthcare System showed a 26\% mean increase in serum creatinine within 3 months among those with an elevated serum creatinine when starting treatment. ${ }^{9}$ Following initiation of an ACEI or ARB, an increase in serum creatinine less than $30 \%$ that subsequently stabilizes is considered acceptable ${ }^{10}$ although experts agree that monitoring serum creatinine levels is a standard of care for these patients. ${ }^{11}$

Recognizing that annual monitoring can reduce preventable adverse drug events, the National Committee on Quality Assurance developed a Healthcare Effectiveness Data and Information Set (HEDIS) performance measure in 2006 for annual monitoring of serum potassium and creatinine for patients on ACEIs or ARBs. ${ }^{12}$ Lack of annual laboratory monitoring may result in delayed diagnosis of clinically important sequelae, yet studies have found that a substantial number of patients do not receive the recommended laboratory tests. One study of several health care delivery systems found that $32 \%$ of prevalent ACEI or ARB users did not receive both recommended laboratory tests, ${ }^{13}$ while another study of ACEI users found that $32 \%$ and $38 \%$ did not receive a creatinine and a potassium test, respectively. ${ }^{14}$ Potential reasons for missing recommended laboratory tests include patient, physician, and systemslevel factors. Quantitative analyses have shown laboratory monitoring to be less likely in younger individuals, in those taking fewer concomitant medications, and in patients with lower health care utilization. ${ }^{13,15,16}$

Following the introduction of the HEDIS performance measure, our health care system implemented new processes to routinely remind patients about laboratory testing. For this study, we aimed to assess the frequency of annual laboratory monitoring by patients on ACEIs and ARBs, as well as contributory factors for lack of monitoring. We also conducted interviews with patients to obtain their insights into facilitators of and barriers to recommended laboratory test monitoring, with the goal of refining our health system's processes to ensure appropriate laboratory monitoring.

\section{Methods}

We conducted a mixed-methods study with a convergent design, combining findings from a retrospective cohort study using electronic health record (EHR) data and individual patient interviews. The study took place within Kaiser Permanente Southern California (KPSC), a large, integrated health system serving 4.7 million racially and socioeconomically diverse members across 234 medical offices. KPSC operates its own pharmacies and laboratories and began implementing its EHR in 2006. It also has an outpatient safety program (the SureNet program), an electronic clinical surveillance system that proactively scans for a variety of issues, including needed laboratory testing for patients on ACEIs or ARBs. ${ }^{17,18}$ Each year, patients who are due for (and have not yet completed) annual laboratory testing are mailed reminder letters.

Our study protocol was reviewed and approved by the KPSC Institutional Review Board (IRB \#10925). A waiver for written informed consent was obtained for the quantitative portion of the study, and verbal consent was approved for patient interviews in conjunction with a written document that covered the elements of informed consent.

\section{STUDY POPULATION}

We included adult KPSC members who were aged 21 years and older, who had at least 180 treatment days of an ACEI or ARB in 2015 (new or prevalent medication users), and who had continuous health plan membership that included a continuous pharmacy benefit (1 administrative gap of $\leq 45$ days was allowed) in the previous year. Treatment days were defined as the actual number of calendar days covered with an ACEI and/or ARB in 2015. For patients who switched from an ACEI to an ARB or vice versa, the combined number of days covered was used to assess whether the patient met the 180 treatment days criterion. 
Inclusion criteria were selected to align with the 2015 HEDIS Annual Monitoring for Patients on Persistent Medications (MPM) criteria. ${ }^{19}$ HEDIS used 18 years as the minimum age; however, our study used 21 years as the minimum age to align with the definition of an adult by federal funding agencies when our study began.

For individual interviews, English-speaking patients were selected using a stratified sampling approach to obtain diverse perspectives. We aimed for $20 \%$ of interviews to be with patients who obtained the recommended laboratory tests following a SureNet reminder letter (10\% from rapid responders, $10 \%$ from eventual responders) and $80 \%$ from patients who did not complete a laboratory test in 2015 .

\section{KEY OUTCOME}

Following the HEDIS MPM definition, the care gap was defined as a patient not receiving the recommended laboratory monitoring of a serum potassium and a creatinine test in 2015. Patients who received only 1 of the 2 tests were classified as having experienced a care gap. The laboratory tests did not need to occur on the same service date to qualify as receipt of recommended laboratory monitoring. Using patient EHRs, laboratory measures were identified from outpatient settings.

\section{QUANTITATIVE ANALYSES}

Baseline Patient Characteristics. Baseline characteristics of the study population were defined using data from 2014 (ie, 2 months before the study year) unless otherwise specified. These data included sociodemographics (age, sex, race/ethnicity, neighborhood education, and income). We used neighborhood income and neighborhood education, estimated by linking home addresses of KPSC members through geocoding and U.S. Census block data, as indicators of socioeconomic status. Baseline characteristics also included using International Classifications of Diseases, Ninth Revision, Clinical Modification (ICD-9-CM) codes to identify history of chronic kidney disease (in the previous 2 years), hypertension, and Charlson Comorbidity Index score. Type I and type II diabetes mellitus was identified using 1 inpatient or 2 outpatient ICD-9-CM codes or the combination of 1 outpatient code and antihyperglycemic medications. Body mass index, length of continuous KPSC membership before 2015, use of the online patient portal (KP.org), and provider characteristics (age, sex, specialty, the provider associated with the first ACEI/ARB dispense in 2015, and years practicing at KPSC) were also captured.
Statistical Analyses. Summary statistics were calculated for patient and provider characteristics stratified by care gap vs no care gap. Means and SDs were calculated for continuous variables, and percentages were calculated for categorical variables. Risk ratios (RRs) and 95\% CIs for having the care gap were estimated using multivariable robust Poisson regression models with generalized estimating equations to account for within-provider correlation. ${ }^{20-23}$ Variables in the final regression model were included based on a priori interest and included all patient- and provider-level baseline characteristics previously mentioned. Analyses were performed using 2-sided P values in SAS Enterprise Guide 7.1 (SAS Institute) and R version 3.6.0 (R Foundation for Statistical Computing).

\section{QUALITATIVE ANALYSES}

Data Collection. The interview guide was developed by the research team with input from clinical and operational team members and guided by the Theoretical Domains Framework (TDF) and our key research questions and domains. ${ }^{24}$ Although the TDF is a framework for physician factors, many of its 14 domains are also relevant to patients, including knowledge, skills, organizational context, intention, goals, and social influence. ${ }^{25}$

Semistructured interviews were conducted with 25 patients by telephone for 30-60 minutes. Interviews were digitally recorded and professionally transcribed. Data collection and analyses occurred iteratively, enabling rapid quality control checks of transcripts, iterative adjustments to the interview guide, and ongoing assessment to determine when additional data no longer yielded new insights. ${ }^{26-28}$

Qualitative Analyses. Coding and analyses were performed using a team coding approach. A primary (lead) coder assigned structural codes for the primary and secondary questions from the interview guide. Once the initial structural codes were developed, 3 transcripts were selected for team coding using the codebook. During the initial coding phase, the primary and secondary coders independently noted any text that resulted in new emergent codes. The researchers then met to review the structural and emergent codes and developed a consensus on a revised codebook, which was used for future coding processes for all remaining transcripts by the lead coder. In the final stages of analyses, both coders developed a hierarchical summary of key domains, themes, and subthemes. Analyses were performed using ATLAS.ti (ATLAS.ti Scientific Software Development GmbH). 


\section{TABLE 1}

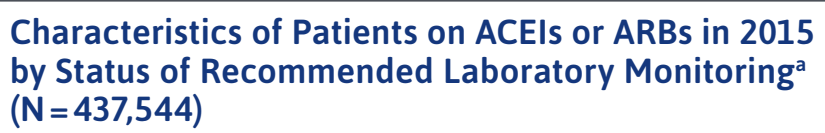

Characteristics of Patients on ACEls or ARBs in 2015 by Status of Recommended Laboratory Monitoring ${ }^{a}$ $(\mathrm{N}=437,544)$

\begin{tabular}{|c|c|c|c|c|}
\hline \multirow[b]{2}{*}{ Characteristic $^{\mathrm{b}}$} & \multicolumn{4}{|c|}{ Recommended laboratory monitoring } \\
\hline & \multicolumn{2}{|c|}{$\begin{array}{c}\text { Received } \\
\text { (No care gap) } \\
\mathrm{n}=398,357 \text { (91.0) }\end{array}$} & \multicolumn{2}{|c|}{$\begin{array}{c}\text { Not received } \\
\text { (Care gap) } \\
\mathrm{n}=39,187(9.0)\end{array}$} \\
\hline Mean age (SD) & 64.3 & $(12.2)$ & 58.4 & $(12.4)$ \\
\hline \multicolumn{5}{|l|}{ Sex } \\
\hline Male & 203,063 & $(51.0)$ & 22,188 & $(56.6)$ \\
\hline Female & 195,294 & $(49.0)$ & 16,999 & $(43.4)$ \\
\hline \multicolumn{5}{|l|}{ Race/ethnicity } \\
\hline Asian or Pacific Islander & 49,295 & $(12.4)$ & 4,402 & $(11.2)$ \\
\hline Black, non-Hispanic & 45,685 & $(11.5)$ & 4,544 & $(11.6)$ \\
\hline Hispanic & 121,572 & $(30.5)$ & 11,322 & $(28.9)$ \\
\hline White, non-Hispanic & 171,162 & $(43.0)$ & 17,189 & $(43.9)$ \\
\hline Multiple/other/unknown & 10,643 & $(2.7)$ & 1,730 & $(4.4)$ \\
\hline \multicolumn{5}{|l|}{ Median neighborhood income } \\
\hline$<\$ 30,000$ & 15,050 & $(3.8)$ & 1,331 & $(3.4)$ \\
\hline$\$ 30,000-\$ 49,999$ & 114,300 & $(28.8)$ & 10,713 & $(27.5)$ \\
\hline$\$ 50,000-\$ 74,999$ & 155,347 & $(39.1)$ & 15,308 & $(39.3)$ \\
\hline$\geq \$ 75,000$ & 112,810 & $(28.4)$ & 11,582 & $(29.7)$ \\
\hline Missing & 850 & $(0.0)$ & 253 & $(0.0)$ \\
\hline \multicolumn{5}{|l|}{ Neighborhood education level } \\
\hline$\leq$ High school & 221,861 & $(55.8)$ & 20,885 & $(53.6)$ \\
\hline Some college or associate degree & 71,054 & $(17.9)$ & 7,475 & $(19.2)$ \\
\hline Bachelor's degree or graduate school & 104,589 & $(26.3)$ & 10,574 & $(27.2)$ \\
\hline Missing & 853 & $(0.0)$ & 253 & $(0.0)$ \\
\hline History of chronic kidney disease & 78,823 & $(19.8)$ & 3,622 & $(9.2)$ \\
\hline History of diabetes mellitus & 163,598 & $(41.1)$ & 8,580 & $(21.9)$ \\
\hline History of hypertension & 264,863 & $(66.5)$ & 19,575 & $(50.0)$ \\
\hline
\end{tabular}

\section{Results}

\section{QUANTITATIVE RESULTS}

Of 437,544 patients prescribed an ACEI or ARB within KPSC during 2015, $9.0 \%$ of patients did not receive both laboratory tests. Table 1 presents patient characteristics stratified by completion of recommended laboratory monitoring tests. Of patients who did not receive both laboratory tests,
84.3\% did not receive either test; $11.3 \%$ only received a serum potassium test; and $4.4 \%$ only received a serum creatinine test.

Patients with a care gap were average (SD) aged 58.4 (2.4) years, and the majority (56.6\%) were male. Patients who completed the recommended laboratory tests were on average (SD) aged 64.3 (12.2) years, and 51.0\% were male. The same proportion of patients
(43.0\%) in both study groups were non-Hispanic White, and the majority lived in census tracts where the most common educational level was high school or less and where annual household incomes were most commonly less than $\$ 75,000$.

A higher proportion of patients with chronic kidney disease $(19.2 \%$ vs $9.2 \%$ ), diabetes mellitus (41.1\% vs $21.9 \%$ ), hypertension ( $66.5 \%$ vs $50.0 \%$ ), and CCI scores of 2 or higher $(45.2 \%$ vs $23.3 \%$ ) received the recommended laboratory tests. Patients who did not have both laboratory tests were enrolled in the KPSC health plan on average (SD) of 3 years less than patients with no care gap (12.9 [12.0] years vs 15.9 [13.6] years). Those who switched from an ACEI to an ARB, or vice versa, in 2015 were more likely to have received the annual recommended monitoring. Characteristics of the patients' providers were similar between the 2 groups (Table 2).

Multivariable analyses (Table 3) were performed on the subset of patients (96.0\%) with complete data on all variables. Increasing age was associated with a reduced risk of not receiving recommended annual laboratory monitoring (RR per 10-year increase $=0.78,95 \% \mathrm{CI}=0.77-0.79$ ), while females were at increased risk of not receiving laboratory monitoring $(\mathrm{RR}=1.10,95 \% \mathrm{CI}=1.08-1.12)$. Asian $(\mathrm{RR}=0.87,95 \% \mathrm{CI}=0.84-0.90) \quad$ and Hispanic (RR=0.91, 95\% CI =0.89-0.94) patients had a reduced risk of missing both tests. A lower risk of a care gap was also observed for patients with diabetes mellitus ( $\mathrm{RR}=0.62$, 95\% CI $=0.60-0.64)$; hypertension $(\mathrm{RR}=0.73,95 \% \mathrm{CI}=0.71-0.74)$; and a CCI score of $1(\mathrm{RR}=0.77,95 \% \mathrm{CI}=0.75-$ $0.79)$ or at least $2(\mathrm{RR}=0.62,95 \%$ $\mathrm{CI}=0.60-0.64)$.

Patients who switched medication classes during the calendar year also had a reduced risk of missing recommended laboratory tests $(\mathrm{RR}=0.53$, 


\section{TABLE 1} Characteristics of Patients on ACEIs or ARBs in 2015
by Status of Recommended Laboratory Monitoring
$(\mathrm{N}=437,544)$ (continued)

\begin{tabular}{|c|c|c|}
\hline \multirow[b]{2}{*}{ Characteristic $^{\mathrm{b}}$} & \multicolumn{2}{|c|}{ Recommended laboratory monitoring } \\
\hline & 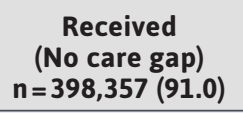 & $\begin{array}{c}\text { Not received } \\
\text { (Care gap) } \\
n=39,187(9.0)\end{array}$ \\
\hline \multicolumn{3}{|l|}{ Charlson Comorbidity Index score } \\
\hline 0 & $120,789 \quad(30.3)$ & $21,079 \quad(53.8)$ \\
\hline 1 & $97,537 \quad(24.5)$ & 8,977 \\
\hline$\geq 2$ & $180,031 \quad(45.2)$ & $9,131 \quad(23.3)$ \\
\hline \multicolumn{3}{|l|}{$\mathrm{BMI}, \mathrm{kg} / \mathrm{m}^{2}$} \\
\hline Normal/underweight $<25$ & $68,134 \quad(17.1)$ & 5,909 \\
\hline Overweight 25 to $<30$ & $100,135 \quad(25.1)$ & 10,364 \\
\hline Obese 30 to $<35$ & $84,675 \quad(21.3)$ & 8,852 \\
\hline Obese $\geq 35$ & $132,268 \quad(33.2)$ & 12,543 \\
\hline Unknown & 13,145 & 1,519 \\
\hline Mean years (SD) of continuous KPSC membership & $15.9 \quad(13.6)$ & $(12.0)$ \\
\hline Used online KPSC patient portal & $270,171 \quad(67.8)$ & $27,901 \quad(71.2)$ \\
\hline Switched medication classes & 34,019 & 1632 \\
\hline \multicolumn{2}{|c|}{$\begin{array}{l}\text { aRecommended laboratory monitoring = serum potassium and serum creatinine tests completed in } 2015 . \\
\text { 'Data shown are } n(\%), \text { unless otherwise indicated. }\end{array}$} & completed in 2015 . \\
\hline
\end{tabular}

95\% CI $=0.51-0.56$ ), as well as those with a cardiologist ( $\mathrm{RR}=0.81,95 \%$ $\mathrm{CI}=0.73-0.90)$ or a nephrologist $(\mathrm{RR}=0.60,95 \% \mathrm{CI}=0.52-0.69)$ as the prescribing provider of the ACEI or ARB. The prescribing provider sex, years practicing at KPSC, provider type, years of continuous enrollment in KPSC before 2015, and online portal use were not meaningfully associated with lack of recommended monitoring (Table 3).

\section{QUALITATIVE RESULTS}

We interviewed 25 patients who were previously sent reminder letters about their laboratory tests: 3 completed the recommended laboratory tests within 30 days of the reminder letter; 2 completed after 30 days; and 20 did not complete the recommended laboratory tests. Patients were consistently able to identify why their medication was prescribed (eg, for hypertension) and were aware of the need to monitor for drug effectiveness (eg, via regular blood pressure checks). In contrast, several patients were unaware of the importance and need for annual laboratory monitoring for side effects from ACEIs or ARBs (Table 4).

Patients reported receiving some information regarding the medications from their primary care provider and some from their pharmacist. Generally, they wanted more information on the purpose of laboratory tests, their importance, and how to interpret results explained in lay terms. Patients often reported that the primary reason for using the online patient portal was to check laboratory test results. However, several patients mentioned they used outside websites to help them understand the laboratory results and wanted more direction for actionable steps to reduce sequelae if they had an abnormal laboratory result.

Although patients generally perceived themselves as proactive and engaged in their care, they believed that lack of motivation might contribute to other patients who miss or delay laboratory tests. At the same time, patients felt that if the importance and purpose of laboratory tests were explained better, most patients would make a greater effort to ensure their completion. Some patients noted that fear of results could delay completion of indicated laboratory tests.

Scheduling issues, the location of laboratories, parking, wait times, and copays were often identified as barriers to obtaining recommended laboratory tests but were not typically viewed as insurmountable challenges or directly linked to missed/delayed tests. Fasting was a perceived barrier despite being unnecessary for serum potassium and creatinine laboratory tests, indicating the need to explicitly communicate this to patients. Patients also might misinterpret acceptable blood pressure readings as an indication that laboratory monitoring is unnecessary, given they were unclear about the difference between monitoring for drug effectiveness vs drug side effects.

Repeated reminders about laboratory tests were generally appreciated, even if the amount of correspondence could be overwhelming. Different patients preferred different contact methods (eg, mailed letter, text message, recorded phone call, or personal phone call). When asked specifically about the SureNet program, which identified patients using ACEIs or 


\section{TABLE 2 Prescribing Provider Characteristics by Patient Status of Recommended Laboratory Monitoring $(\mathrm{N}=437,544)$}

\begin{tabular}{|c|c|c|}
\hline \multirow[b]{2}{*}{ Provider characteristic } & \multicolumn{2}{|c|}{$\begin{array}{c}\text { Recommended laboratory } \\
\text { monitoring }\end{array}$} \\
\hline & $\begin{array}{c}\text { Received } \\
\text { (No care gap) } \\
n=398,357\end{array}$ & $\begin{array}{c}\text { Not received } \\
\text { (Care gap) } \\
\mathrm{n}=39,187\end{array}$ \\
\hline Mean age (SD) & $46.1(8.7)$ & $46.1 \quad(8.7)$ \\
\hline \multicolumn{3}{|l|}{ Sex, n (\%) } \\
\hline Male & $233,459(58.9)$ & $23,846(61.1)$ \\
\hline Female & $163,010(41.1)$ & $15,173(38.9)$ \\
\hline \multicolumn{3}{|l|}{ Provider specialty, n (\%) } \\
\hline Primary care & $361,888(90.8)$ & $36,328(92.7)$ \\
\hline Cardiology & $10,483 \quad(2.6)$ & 738 (1.9) \\
\hline Nephrology & $7,335 \quad(1.8)$ & $303 \quad(0.8)$ \\
\hline Other & $18,651 \quad(4.7)$ & $1,818 \quad(4.6)$ \\
\hline \multicolumn{3}{|l|}{ Patient provider type, n (\%) } \\
\hline Own PCP & $320,956(81.0)$ & $31,807(81.5)$ \\
\hline Other PCP & $50,411(12.7)$ & $5,240(13.4)$ \\
\hline Other medical doctor & $16,251 \quad(4.1)$ & $1,167 \quad(3.0)$ \\
\hline Other specialist & $8,844 \quad(2.2)$ & $806 \quad(2.1)$ \\
\hline Missing & $1,895 \quad(0.0)$ & 167 (0.0.) \\
\hline Years practicing at KPSC, mean (SD) & $12.6(8.7)$ & $12.4 \quad(8.7)$ \\
\hline
\end{tabular}

ARBs and generated letters reminding them that they were due for laboratory tests, patients generally expressed high regard for the program.

\section{Discussion}

Nearly $10 \%$ of patients did not receive recommended laboratory test monitoring for medication-related adverse drug events within our integrated health care delivery system. This occurred despite a program to proactively send reminders to patients using ACEIs or ARBs about required monitoring. Given the large number of patients using these medications, and the recent expansion of prescribed antihypertensive medications in the US adult population, ${ }^{29}$ more patients will be at risk of adverse drug events, underscoring the importance of preventive laboratory monitoring.

We found that despite the reminder letters about pending laboratory test orders, patients did not seem to directly connect these tests to their ACEI or ARB medications. Moreover, patients consistently reported not knowing that the laboratory tests were intended to monitor for drug side effects and expressed confusion over the difference between blood pressure monitoring and tests for side effects. Although patients reported a number of logistical barriers, they did not describe them as the primary barrier or factor that would prevent laboratory monitoring if they had understood the importance of testing.

In contrast to our results, a mixed-methods study among patients in a large, multispecialty group practice who were prescribed medications requiring laboratory monitoring found that most patients were knowledgeable about the reason (eg, preventing side effects) for their laboratory tests. ${ }^{15}$ Our study was limited to patients taking ACEIs or ARBs, while this study included 34 medications and included chemotherapy agents and blood thinners, which require more frequent laboratory testing.

The multivariable analyses in our study show that patients who are older and have more comorbidities are less likely to have a care gap in laboratory monitoring. One possible explanation is that the creatinine and potassium laboratory orders are pending in the laboratory system, so if these patients go to the laboratory for any other reason, these tests will be performed. Another reason for fewer care gaps in older patients may reflect the greater seriousness and increased risk of side effects for them or the increased number of visits and providers involved in their health care.

In a study of 167,721 patients with diabetes in the KPSC health care system, we found that overweight and obese individuals had higher odds of being screened for hemoglobin A1c and lipids according to HEDIS quality of care measures for diabetes. ${ }^{30}$ This finding could have resulted from health care providers having more opportunities to order screening tests. More specifically, increasing age, a diagnosis of diabetes mellitus, and hypertension were factors significantly less likely to be associated with missing the recommended laboratory tests.

A review of the current knowledge of hyperkalemia associated with ACEI and ARB therapies noted the potential risks for hyperkalemia, such as increasing age, diabetes mellitus, and kidney diseases ${ }^{31}$ which increase the importance of completing laboratory tests to identify drug side effects among these at-risk patients. In addition, patients with a cardiologist or a nephrologist (vs a primary care provider) as the prescribing provider of the ACEIs or ARBs were at a reduced risk of missing the recommended laboratory monitoring.

In a study that assessed noncompletion of ordered laboratory tests among 27,802 patients prescribed at least 1 of 34 chronic disease medications in a large multispecialty group practice, the odds of test noncompletion were higher 


\section{TABLE 3 Multivariable Risk Factors Associated With not Receiving Recommended Laboratory Monitoring $(\mathrm{N}=420,730)$}

\begin{tabular}{|c|c|c|c|c|c|}
\hline Characteristic & $\begin{array}{l}\text { Relative } \\
\text { risk }\end{array}$ & $95 \% \mathrm{Cl}$ & Characteristic & $\begin{array}{l}\text { Relative } \\
\text { risk }\end{array}$ & $95 \% \mathrm{Cl}$ \\
\hline Age (10-year increments) & 0.78 & $(0.77-0.79)$ & \multicolumn{3}{|l|}{ BMI } \\
\hline \multicolumn{3}{|l|}{ Sex } & Normal/underweight $<25$ & 1.00 & Referent \\
\hline Male & 1.00 & Referent & Overweight 25 to $<30$ & 0.95 & $(0.93-0.98)$ \\
\hline Female & 1.10 & $(1.08-1.12)$ & Obese 30 to $<35$ & 0.98 & $(0.95-1.01)$ \\
\hline \multicolumn{3}{|l|}{ Race/ethnicity } & Obese $\geq 35$ & 0.95 & $(0.92-0.98)$ \\
\hline $\begin{array}{l}\text { Non-Hispanic White } \\
\text { Non-Hispanic Black }\end{array}$ & 1.00 & $\begin{array}{l}\text { Reterent } \\
(0.97-1.04)\end{array}$ & Years of continuous KPSC membership & 0.99 & $(0.99-1.00)$ \\
\hline Hispanic & 0.91 & $(0.89-0.94)$ & Use online KPSC portal & 0.96 & $(0.94-0.99)$ \\
\hline Asian & 0.87 & $(0.84-0.90)$ & Switched medication classes & 0.53 & $(0.51-0.56)$ \\
\hline Other & 1.15 & $(1.10-1.21)$ & Provider age (10-year increments) & 1.06 & $(1.03-1.09)$ \\
\hline \multicolumn{3}{|l|}{ Median neighborhood income } & \multicolumn{3}{|l|}{ Provider sex } \\
\hline$<\$ 30,000$ & 1.00 & Referent & Male & 1.00 & Referent \\
\hline$\$ 30,000-\$ 49,999$ & 1.01 & $(0.95-1.07)$ & Female & 1.04 & $(1.01-1.08)$ \\
\hline$\$ 50,000-\$ 74,999$ & 1.00 & $(0.95-1.07)$ & \multicolumn{3}{|l|}{ Provider specialty } \\
\hline$\geq \$ 75,000$ & 0.99 & $(0.93-1.05)$ & Primary care & 1.00 & Referent \\
\hline \multicolumn{3}{|l|}{ Neighborhood education level } & Cardiology & 0.81 & $(0.73-0.90)$ \\
\hline Some college or associate degree & 1.05 & $(1.02-1.08)$ & Nephrology & 0.60 & $(0.52-0.69)$ \\
\hline Bachelor's degree or graduate school & 1.05 & $(1.02-1.09)$ & Other & 0.95 & $(0.88-1.03)$ \\
\hline History of chronic kidney disease & 0.97 & $(0.93-1.01)$ & \multicolumn{3}{|l|}{ Patient provider type } \\
\hline History of diabetes mellitus & 0.62 & $(0.60-0.64)$ & Own PCP & 1.00 & Referent \\
\hline History of hypertension & 0.73 & $(0.71-0.74)$ & Other PCP & 1.07 & $(1.03-1.11)$ \\
\hline \multicolumn{3}{|l|}{ Charlson Comorbidity Index score } & Other medical doctor & 1.02 & $(0.94-1.10)$ \\
\hline 0 & 1.00 & Referent & Other specialist & 1.02 & $(0.91-1.13)$ \\
\hline 1 & 0.77 & $(0.75-0.79)$ & \multirow{2}{*}{$\begin{array}{l}\text { Years practicing at KPSC } \\
\text { (5-year increments) }\end{array}$} & \multirow{2}{*}{0.99} & \multirow{2}{*}{$(0.97-1.00)$} \\
\hline$\geq 2$ & 0.62 & $(0.60-0.64)$ & & & \\
\hline
\end{tabular}

when a primary care provider vs a specialist ordered the test. ${ }^{15}$ The findings from both studies suggest that specialists may be more likely to emphasize laboratory monitoring, given that they are likely treating a higher-risk population.

In our setting, with reminders sent to patients about testing, a lower percentage missed recommended testing compared with previous reports (10\% vs $\sim 30 \%) .{ }^{12,14,32}$ However, during our qualitative interviews, several patients noted the importance of reducing medical jargon when communicating with patients. With this in mind, our team developed an education sheet for patients to read when they picked up medications, which clearly explained the need for monitoring drug effectiveness and drug side effects (Supplementary Material, available in online article). Several patients mentioned that they had conversations with their pharmacists, as well as their prescribing providers, indicating that this type of educational sheet could be provided by providers and pharmacists to educate and remind patients of the need for monitoring.

\section{LIMITATIONS}

There are several study limitations. Our analysis was conducted within a single, integrated health care system with an active electronic surveillance program focused on 


\section{TABLE 4 Knowledge, Facilitators, and Barriers Among Patients Receiving ACEls or ARBs}

\section{Topic}

Lack of knowledge about annual lab monitoring for side effects

Lack of knowledge about annual lab monitoring for side effects

Lack of knowledge about annual lab monitoring for side effects

Desire for information/knowledge

Desire for information/knowledge

for information/knowledge "

Patient portal

Personal motivation/family support

Personal motivation/family support

Fear of results/criticism

Logistical barriers

Logistical barriers

Logistical barriers

Misconceptions about fasting

Repeated reminders from KPSC

Repeated reminders from KPSC

Reminder- SureNet

\section{Representative Quotes}

"I have blood drawn a couple times a year...but I've never been made aware that drawing the blood is related to testing my hypertension."

No, there's never...been an indication that [the] high blood pressure medicine I've been taking...that there's a reason to get bloodwork done because of that."

"Why would I get a lab test for it anyway? When you go in and they take your blood pressure, you would see if it's working for you when your blood pressure is lower, right? So...[a] lab hasn't even been on my radar like to ask, 'don't I need a lab test to make sure of whatever you're looking for to put me on this lisinopril?' I've never asked. They never said."

"The fact that my doctor didn't tell me is a [cause for] concern because that information should be told... now that I know, I probably would take [the lab test]."

"Oh, we do get those [after visit summaries]. You're given a whole list...your symptoms, all your conditions.. all the medications that you're taking. The first time anything is prescribed, it's described to you [by the pharmacy], what it's for, how to use it, verbally, and then they ask you if you have any questions. You get multiple reinforcements, which is particularly good because sometimes you don't hear everything lyour doctor is] saying. You don't process everything they're saying."

"A little blurb for each test, 'Okay, this is what this test is -' I mean, besides, like, the normal range...maybe a little thing saying, 'We test this for this reason...."

"Yes, [I get lab tests completed] consistently every year. I just put it in my calendar. I make sure that I'm consistent also with my annual checkup because I'm monitoring...my health."

"I get a lot of things in the mail...from Kaiser. I give it to my wife. And I tell her, look, read this stuff. And if any of it's important, let me know."

"I have a personality that makes me sometimes live in denial...what I don't know won't hurt me."

"My only issue is...having an extra time or opening a Saturday clinic just for labs, or if they do...making somebody who needs to do bloodwork more aware of that because they don't [advertise that]."

"Not every Kaiser you have to pay for parking, but parking does become an issue....[but you] learn how to work the time of the day to get around that."

"For my lab, [the copay amount] changed recently... And yes, it would be pretty hard if you had to do it a lot."

"But it also has to do with the fact that...you need to fast. And a lot of the times I'm up, you know, 6:00, 7:00 am in the morning. I'm not going to...fast all day and all night until the time I finally can make it over to the hospital to get my lab work done."

"[Send me] a weekly text message until obviously I'm constantly reminded until the point to where it's like, okay, enough, I'm coming in....you can't really bother somebody enough when it comes to [their health]."

"And sometimes it can be a little nagging...when Kaiser calls you...a couple of times a week...l admit that. But overall...it's for a good thing."

"It's good....there is someone in the background that's monitoring, making sure that things get done... things are easy to fall through the crack, and [doctors are] busy."

$A C E I=$ angiotensin-converting enzyme inhibitor; $A R B=$ angiotensin receptor blocker; $K P S C=$ Kaiser Permanente Southern California .

outpatient safety. The lack of a similar surveillance program may be associated with a higher incidence in care gaps in other settings. Although KPSC has an outpatient safety net program, it does not measure the incidence or identify causes of care gaps, thereby limiting comparisons across populations of different sizes. Furthermore, there may be important disparities in care by insurance status, age, sex, or race/ethnicity that were not evident within our relatively equal-access system.
Our qualitative results may have been influenced by social desirability bias which would have overestimated the positive responses to questions pertaining to knowledge and facilitators of laboratory monitoring. Finally, the qualitative results are not representative of the study population and thus may not be generalizable.

Strengths of our study include the pairing of quantitative and qualitative data to identify the frequency of care gaps, contributing factors, and potential ways to reduce them. In addition, within our integrated delivery system, we were 
able to link a wide variety of patient data and provider characteristics.

\section{Conclusions}

We found that $9 \%$ of patients using ACEIs or ARBs lacked the recommended laboratory monitoring that is important for prompt identification of side effects and prevention of serious sequelae. Given the increasing number of patients using ACEIs and ARBs, monitoring strategies, including an electronic clinical surveillance system, could show a substantial benefit in reducing adverse drug events by increasing compliance with recommended laboratory testing. Future studies to better understand the effect of missed monitoring are also needed.

\section{DISCLOSURES}

This project was supported by grant number R01HS024437 from the Agency for Healthcare Research and Quality. The funder had no role in the design of the study; collection, analyses, or interpretation of the data, or decision to submit this manuscript for publication. Harrison, Reynolds, Hahn, Munoz-Plaza, Yi, Fischer, Luong, Sim, Brettler, Handler, and Mittman are employees of the Southern California Permanente Medical Group. Danworth was employed by the Southern California Permanente Medical Group at the time of this study. Singh was partially supported by the Houston VA HSR\&D Center for Innovations in Quality, Effectiveness and Safety (CIN13-413). Reynolds reports grants from Novartis, Amgen Inc., and Vital Strategies, Resolve to Save Lives, unrelated to this work. Yi reports grants from Novartis unrelated to this work. Kanter has nothing to disclose.

\section{ACKNOWLEDGMENTS}

The authors thank the patients of Kaiser Permanente for helping to improve care through the use of information collected through our electronic health record systems. We also thank the KPSC physicians, and KPSC's Quality and Clinical Analysis and SureNet teams for their participation and assistance.

\section{REFERENCES}

1. Whelton PK, Carey RM, Aronow WS, et al. 2017 ACC/AHA/AAPA/ABC/ACPM/ AGS/APhA/ASH/ASPC/NMA/PCNA guideline for the prevention, detection, evaluation, and management of high blood pressure in adults: a report of the American College of Cardiology/American Heart Association Task Force on Clinical Practice Guidelines. J Am Coll Cardiol. 2018;71(19):e127-e248.

2. Yancy CW, Jessup M, Bozkurt B, et al. 2017 ACC/AHA/HFSA focused update of the 2013 ACCF/AHA guideline for the management of heart failure. J Card Fail. 2017;70(6):776-803.

3. National Kidney Foundation. KDOQI clinical practice guideline for diabetes and CKD: 2012 update. Am J Kidney Dis. 2012;60(5):850-86.

4. Aitken M, Kleinrock M. Medicine use and spending in the U.S.: a review of 2018 and outlook to 2023. The IQVIA Institute. May 9, 2019. Accessed November 13, 2021. https://www.iqvia.com/ insights/the-iqvia-institute/reports/ medicine-use-and-spending-in-the-us-areview-of-2018-and-outlook-to-2023

5. Ahuja TS, Freeman D Jr, Mahnken JD, Agraharkar M, Siddiqui M, Memon A. Predictors of the development of hyperkalemia in patients using angiotensin-converting enzyme inhibitors. Am J Nephrol. 2000;20(4):268-72.

6. Palmer BF. Angiotensin-converting enzyme inhibitors and angiotensin receptor blockers: what to do if the serum creatinine and/or serum potassium concentration rises. Nephrol Dial Transplant. 2003;18(10):1973-75.

7. Reardon LC, Macpherson DS.

Hyperkalemia in outpatients using angiotensin-converting enzyme inhibitors. How much should we worry? Arch Intern Med. 1998;158(1):26-32.

8. Chang AR, Sang Y, Leddy J, et al. Antihypertensive medications and the prevalence of hyperkalemia in a large health system. Hypertension. 2016;67(6):1181-88.
9. Jackevicius CA, Wong J, Aroustamian I, Gee M, Mody FV. Rates and predictors of ACE inhibitor discontinuation subsequent to elevated serum creatinine: a retrospective cohort study. BMJ Open. 2014;4(8):e005181.

10. Bakris GL, Weir MR. Angiotensinconverting enzyme inhibitor-associated elevations in serum creatinine: is this a cause for concern? Arch Intern Med. 2000;160(5):685-93.

11. Knight EL, Avorn J. Quality indicators for appropriate medication use in vulnerable elders. Ann Intern Med. 2001;135 (8 Pt 2):703-10.

12. Raebel MA, McClure DL, Simon SR, et al. Laboratory monitoring of potassium and creatinine in ambulatory patients receiving angiotensin converting enzyme inhibitors and angiotensin receptor blockers. Pharmacoepidemiol Drug Saf. 2007;16(1):55-64.

13. Raebel MA, McClure DL, Chan KA, et al. Laboratory evaluation of potassium and creatinine among ambulatory patients prescribed spironolactone: are we monitoring for hyperkalemia? Ann Pharmacother. 2007;41(2):193-200.

14. Hurley JS, Roberts M, Solberg LI, et al. Laboratory safety monitoring of chronic medications in ambulatory care settings. J Gen Intern Med. 2005;20(4):331-33.

15. Fischer SH, Field TS, Gagne SJ, et al. Patient completion of laboratory tests to monitor medication therapy: a mixed-methods study. J Gen Intern Med. 2013;28(4):513-21.

16. Moffet HH, Parker MM, Sarkar U, et al. Adherence to laboratory test requests by patients with diabetes: the Diabetes Study of Northern California (DISTANCE). Am J Manag Care. 2011;17(5):339-44.

17. Danforth KN, Smith AE, Loo RK, Jacobsen SJ, Mittman BS, Kanter MH. Electronic clinical surveillance to improve outpatient care: diverse applications within an integrated delivery system. EGEMS (Wash DC). 2014;2(1):1056. 
18. Sim JJ, Rutkowski MP, Selevan DC, et al. Kaiser Permanente Creatinine Safety Program: a mechanism to ensure widespread detection and care for chronic kidney disease. Am J Med. 2015;128(11):1204-11.e1201.

19. National Committee for Quality Assurance. HEDIS 2015: Healthcare Effectiveness Data and Information Set. Vol 2. Washington, DC; 2015.

20. Carter RE, Lipsitz SR, Tilley BC. Quasi-likelihood estimation for relative risk regression models. Biostatistics. 2005;6(1):39-44.

21. McNutt LA, Wu C, Xue X, Hafner JP. Estimating the relative risk in cohort studies and clinical trials of common outcomes. Am J Epidemiol. 2003;157(10):940-43.

22. Traissac P, Martin-Prével Y, Delpeuch F, Maire B. [Logistic regression vs other generalized linear models to estimate prevalence rate ratios]. Rev Epidemiol Sante Publique. 1999;47(6):593-604.
23. Zou G. A modified Poisson regression approach to prospective studies with binary data. Am J Epidemiol. 2004;159(7):702-06.

24. Bradley EH, Curry LA, Devers KJ. Qualitative data analysis for health services research: developing taxonomy, themes, and theory. Health Serv Res. 2007;42(4):1758-72.

25. Michie S, Johnston M, Abraham C, Lawton R, Parker D, Walker A. Making psychological theory useful for implementing evidence based practice: a consensus approach. Qual Saf Health Care. 2005;14(1):26-33.

26. Francis JJ, Johnston M, Robertson C, et al. What is an adequate sample size? Operationalising data saturation for theory-based interview studies. Psychol Health. 2010;25(10):1229-45.

27. Guest G, Bunce A, Johnson L, Methods JF. How many interviews are enough?: an experiment with data saturation and variability. Field Methods. 2006;18:59-82.
28. Saunders B, Sim J, Kingstone T, et al. Saturation in qualitative research: exploring its conceptualization and operationalization. Qual Quant. 2018;52(4):1893-07.

29. Muntner P, Carey RM, Gidding S, et al. Potential US population impact of the 2017 ACC/AHA high blood pressure guideline. Circulation. 2018;137(2):109-18.

30. Ryan DS, Coleman KJ, Lawrence JM, Harrison TN, Reynolds K. Optimal management of diabetes among overweight and obese adults. Am J Manag Care. 2014;20(1):e1-7.

31. Raebel MA. Hyperkalemia associated with use of angiotensin-converting enzyme inhibitors and angiotensin receptor blockers. Cardiovasc Ther. 2012;30(3):e156-166.

32. Raebel MA, Lyons EE, Andrade SE, et al. Laboratory monitoring of drugs at initiation of therapy in ambulatory care. J Gen Intern Med. 2005;20(12):1120-26. 